\title{
Gestione del carcinoma epatocellulare: le linee guida internazionali
}

\section{Management of hepatocellular carcinoma: international guidelines}

\section{Luigi Fenoglio $^{a, *}$, Elisabetta Castagna ${ }^{a}$, Cristina Serraino ${ }^{a}$, Adele Cardellicchio ${ }^{a}$, Fulvio Pomero ${ }^{a}$, Christian Bracco ${ }^{a}$, Maurizio Grosso ${ }^{b}$}

\author{
a SC Medicina Interna (Direttore: dott. Luigi Fenoglio), ASO "Santa Croce e Carle", Cuneo; I Scuola di Specializzazione \\ in Medicina Interna, Università degli Studi di Torino \\ ${ }^{\mathrm{b}}$ SC Radiodiagnostica (Direttore: dott. Maurizio Grosso), ASO "Santa Croce e Carle", Cuneo
}

Ricevuto il 29 settembre 2008; accettato il 6 ottobre 2008

disponibile online il 13 luglio 2009

\section{KEYWORDS \\ Hepatocellular \\ carcinoma; \\ Surveillance; \\ Management.}

\begin{abstract}
Summary Hepatocellular carcinoma (HCC) is a major health problem worldwide. The incidence of HCC is increasing in Europe and in the United States. HCC is currently the leading cause of death among cirrhotic patients. Cirrhosis is the strongest and the most common risk factor for $\mathrm{HCC}$. Surveillance for HCC is widely practiced and can be recommended for certain at-risk groups. Among serological screening test, alpha-fetoprotein (AFP) is the best known (cut-off $20 \mathrm{ng} / \mathrm{mL}$, sensitivity $60 \%$, specificity $91 \%$ ). The radiological screening test most widely used is ultrasonography (sensitivity $65-80 \%$, specificity $>90 \%$ ). The tests used to diagnose HCC include radiology, biopsy and AFP. Detection of hepatic mass within a cirrhotic liver is highly suspicious of HCC. If AFP is greater than $200 \mathrm{ng} / \mathrm{mL}$ and the radiological appearance of the mass is suggestive for HCC, the likelihood that the lesion is HCC is high. The Barcelona-Clinic-Liver-Cancer staging system identifies patients with early HCC who may benefit from curative therapies, those at intermediate or advanced disease stage who may benefit from palliative treatments, as well as those at end-stage with a very poor life expectancy. Today many patients are diagnosed at an early stage. The therapies that offer a high rate of complete responses and potential cure are surgical resection, transplantation and percutaneous ablation. Among non-curative therapies the only one that has been shown to positively impact survival is transarterial chemoembolization. Several steps have to be taken to improve effectiveness of HCC therapy. These include patient education on risk factors for $\mathrm{HCC}$ and implementation of screening programs, increasing the number of patients diagnosed in early stage.

(c) 2009 Elsevier Srl. All rights reserved.
\end{abstract}

\footnotetext{
* Corrispondenza: SC Medicina Interna, ASO Santa Croce e Carle, via Michele Coppino 26 - 12100 Cuneo.
}

E-mail: medicina.interna@ospedale.cuneo.it (L. Fenoglio). 


\section{Introduzione}

Le raccomandazioni di seguito riportate forniscono un approccio evidence-based a diagnosi, stadiazione e trattamento del carcinoma epatocellulare (HCC). Esse vengono proposte in base a:

- revisione e analisi dei dati recenti riportati in letteratura;

- linee guida internazionali (American Association for the Study of Liver Diseases, AASLD; European Association for the Study of the Liver, EASL).

La qualità dell'evidenza a supporto delle singole raccomandazioni è definita dalle categorie riportate in tabella 1.

\section{Epidemiologia}

L'HCC costituisce la più severa complicanza delle malattie epatiche croniche. Il processo inizia con un insulto epatico acuto o subacuto, che progredisce in fibrosi parenchimale e quindi in cirrosi in un arco di tempo di circa 20-30 anni. La cirrosi epatica costituisce il più importante fattore predisponente allo sviluppo di HCC [1]; raramente il tumore si manifesta in assenza di cirrosi [2].

L'HCC rappresenta il 6\% delle neoplasie, con un'incidenza mondiale pari a 0,5 milioni di nuovi casi/anno [3]; è la principale causa di morte nella cirrosi [4].

Risultano notevoli discrepanze geografiche nella distribuzione della neoplasia in relazione alla differente esposizione ai fattori di rischio. L'incidenza di HCC è maggiore nei Paesi in via di sviluppo (>100 casi per 100.000 abitanti/ anno) [5], caratterizzati da elevata incidenza di infezione da virus dell'epatite B (HBV) (Sud-Est asiatico, Africa subsahariana).

Recentemente si è assistito all'aumento di incidenza dell'HCC in aree endemiche per infezione da virus dell'epatite C (HCV) (Giappone, Italia e Stati Uniti) [5,6]. L'epidemia di epatite $C$ ha avuto inizio negli anni Sessanta e, poiché tra l'infezione e lo sviluppo di cirrosi intercorre un lungo periodo, si assisterà nei prossimi 20 anni a un continuo incremento dell'incidenza di HCC HCV-relato [5,6]. In Italia l'incidenza è attualmente di 12.000 nuovi casi/anno [7,8].

Nelle casistiche americane ed europee $[9,10]$, negli anni Ottanta-Novanta la sopravvivenza dei pazienti affetti da HCC è rimasta stabile. Migliori risultati sono riportati dagli studi condotti in centri di riferimento rispetto agli studi di popolazione: discrepanza correlabile al bias di selezione (i pazienti afferenti ai centri di riferimento sono più frequentemente affetti da tumori non avanzati) e al fatto che, poiché le modalità di trattamento dell'HCC sono molto complesse, può esservi un gap fra lo stato dell'arte e la comune pratica clinica [3].

Tabella 1 Livelli di evidenza secondo il disegno dello studio.

\begin{tabular}{ll}
\hline Grado & Definizione \\
\hline I & Studi randomizzati controllati \\
\hline II & $\begin{array}{l}\text { Studi controllati non randomizzati, } \\
\text { studi caso-controllo, studi di coorte }\end{array}$ \\
\hline III & Opinione di esperti, studi epidemiologici descrittivi \\
\hline
\end{tabular}

\section{Fattori di rischio}

Età avanzata e sesso maschile si associano a una maggiore incidenza di HCC [11]. Tale neoplasia è fino a 4 volte più frequente negli uomini che nelle donne; la massima incidenza si osserva nei Paesi occidentali durante il quinto e sesto decennio di vita e uno o due decenni prima nei Paesi asiatici e africani [3], come conseguenza della maggiore incidenza di infezione neonatale e perinatale da HBV.

Nel seguito si riportano alcuni fattori di rischio per HCC. - HBV: è la causa più comune di HCC nel mondo. Il rischio di HCC nei soggetti con HBV è 5-15 volte superiore rispetto alla popolazione generale; il $10-25 \%$ dei casi di HCC-HBV associati si sviluppa in assenza di cirrosi [8]. I portatori di HBV presentano un rischio basso, ma non trascurabile, di sviluppare HCC [12]; la condizione di portatore viene definita dalla positività dell'HBsAg $>6$ mesi, con HBeAg negativo e anticorpi anti-HBe positivi, in presenza di livelli di HBV DNA $<2.000 \mathrm{UI} / \mathrm{mL}$, di livelli di transaminasi costantemente nella norma e in assenza di epatite alla biopsia epatica. La determinazione della replicazione virale nei soggetti HBsAg positivi è importante per stabilire il rischio di HCC, essendo quest'ultimo direttamente correlato ai valori di viremia [12].

- HCV: il rischio di HCC in pazienti con infezione da HCV è 17 volte superiore rispetto ai non portatori; virtualmente tutti i casi di HCC-HCV associati si sviluppano in presenza di cirrosi. L'incidenza annuale di HCC è pari a 1,0-4,0\% $(0,5 \%$ in pazienti con fibrosi epatica di basso grado; $7,9 \%$ in pazienti con fibrosi di alto grado). La gravità istologica del danno epatico è il fattore che più di ogni altro condiziona il rischio di tumore [12].

- Alcol: la relazione tra etanolo e HCC è ben documentata, con un'associazione di dose-dipendenza nella maggior parte degli studi. La presenza di cirrosi sembra aumentare di 10 volte il rischio di sviluppare HCC nei bevitori cronici (>40-60 g/die di etanolo nell'uomo e $>20-40 \mathrm{~g}$ nella donna per 10-20 anni) [13].

- Aflatossina: è un prodotto del metabolismo dell'Aspergillus flavus, fungo che contamina i cibi in molti Paesi tropicali. Sperimentalmente, è tra i più potenti carcinogeni epatici conosciuti. Nell'HCC l'aflatossina amplificherebbe il rischio determinato dall'infezione da HBV [3].

- Tabacco: i forti fumatori hanno un rischio relativo pari a 1,5-2 di sviluppare HCC [14].

- Emocromatosi ereditaria: il rischio di HCC risulta aumentato [15]; il rischio relativo in tali soggetti è di circa 20.

Per quanto concerne il diabete e la steatosi epatica non alcolica (NAFLD), non vi sono attualmente sufficienti dati di evidenza a causa della mancanza di adeguati studi epidemiologici.

\section{Sorveglianza}

Per "sorveglianza" s'intende l'esecuzione ripetuta di test diagnostici in una popolazione selezionata di soggetti patologici senza sintomi riferibili alla complicanza relata alla patologia di base (per esempio, i portatori di epatopatia a rischio di complicanze quali l'HCC).

Le manifestazioni cliniche di HCC sono tardive; la diagnosi è generalmente posta in stadio avanzato di malattia con 
conseguenti limitate possibilità terapeutiche. La sopravvivenza a 5 anni varia tra lo $0 \%$ e il $10 \%[16,17]$. Un trial randomizzato controllato (RCT) ha evidenziato una prognosi migliore nei soggetti monitorati semestralmente con ecografia epatica e dosaggio sierico dell'alfa-fetoproteina (AFP) [17]. La sorveglianza viene attuata con l'obiettivo di identificare neoplasie di piccole dimensioni, con buone opportunità terapeutiche e un buon rapporto costo/efficacia, evidenziato nella maggior parte degli studi [18].

In letteratura è riportato un recente incremento delle diagnosi precoci di HCC conseguentemente all'implementazione di programmi di sorveglianza per i soggetti a rischio [19].

La decisione di sottoporre a un programma di sorveglianza un paziente è determinata dal rischio di HCC; pur non sussistendo dati di letteratura che indichino a partire da quale grado di rischio sia necessario sottoporre il paziente a sorveglianza, è possibile identificare gruppi di pazienti con associati fattori di rischio.

\section{Epatite virale B}

In accordo con le linee guida AASLD [18] relative a pazienti HBsAg positivi (livello III):

- in caso di anamnesi familiare positiva per HCC primitivo, l'osservazione inizia prima dei 40 anni;

- tutti i soggetti affetti da cirrosi HBV-relata vanno sottoposti a programma di sorveglianza;

- la sorveglianza per HCC è raccomandata negli uomini asiatici di età $>40$ anni e nelle donne asiatiche di età > 50 anni. L'incidenza è minore nel sesso femminile. Negli asiatici in cui l'HBsAg si negativizza dopo un lungo stato di portatore, è raccomandato proseguire la sorveglianza;

- nelle popolazioni africane, la sorveglianza va iniziata a partire dai 20 anni, per l'elevata incidenza di HCC già nel terzo decennio di vita a causa degli alti tassi di infezione neonatale;

- nei soggetti caucasici portatori di HBV, senza evoluzione cirrotica, transaminasi ripetutamente nella norma e ridotta concentrazione di DNA virale $\left(<2.000 \mathrm{UI} / \mathrm{mL}=10^{4}\right.$ coppie $/ \mathrm{mL}$ ), il rischio di HCC è basso e si riduce significativamente nei pazienti che negativizzano l'antigene di superficie. Tuttavia, fattori di rischio aggiuntivi, come l'età avanzata, la coinfezione da HCV o HIV e la coesistenza di altre cause di epatopatie (etanolo), pongono l'indicazione a un programma di sorveglianza;

\section{Epatite virale C}

Il rischio di $\mathrm{HCC}$ è molto elevato in caso di infezione da $\mathrm{HCV}$, essendo 17 volte più alto rispetto agli individui non HCV positivi; in presenza di cirrosi, la sua incidenza sale al 2-8\% per anno [20]. Il passaggio dalla fibrosi alla cirrosi non può essere determinato clinicamente, pertanto, in occasione della conferenza EASL del 2000, è stato stabilito di sottoporre a sorveglianza per HCC tutti i soggetti affetti da HCV con cirrosi o fibrosi epatica avanzata [21].

Non vi sono al momento dati in letteratura inerenti l'utilizzo del FibroScan in programmi di sorveglianza basati sulla correlazione tra entità della fibrosi e rischio di HCC [22].

\section{Coinfezione da HIV}

Una più rapida progressione del danno epatico si verifica in caso di epatite B o C associata a infezione da HIV che, in presenza di cirrosi, aumenta anche il rischio di HCC [23].

\section{Cause non virali di cirrosi epatica}

L'incidenza di HCC in altre forme di epatopatia cronica dipende dai singoli fattori eziopatogenetici.

- La cirrosi alcolica è una condizione significativamente favorente lo sviluppo di HCC, per cui le linee guida AASLD consigliano un programma di sorveglianza.

- Con l'identificazione della NAFLD come causa di cirrosi, in letteratura sono comparsi dati indicanti un aumentato rischio di HCC in tale forma di cirrosi. Sino a quando non sarà nota l'incidenza di HCC in caso di cirrosi dovuta a NAFLD, non sarà possibile consigliare un programma di sorveglianza.

- Nell'emocromatosi familiare la cirrosi espone a un rischio elevato di HCC, con rischio relativo pari a 20 [14] e indicazione al programma di sorveglianza.

- L'incidenza di HCC in IV stadio di cirrosi biliare primitiva è sovrapponibile a quella riscontrabile nella cirrosi HCVrelata, per cui le linee guida AASLD raccomandano un programma di sorveglianza.

- Riguardo alla cirrosi indotta dal deficit di alfa1-antitripsina o dall'evoluzione di un'epatite autoimmune, non sono disponibili dati sufficienti a definire il rischio di HCC.

\section{Ulteriori considerazioni}

Attualmente non sussistono evidenze sufficienti a chiarire se e quanto la terapia con interferone riduca il rischio di HCC in caso di epatite B o C cronica. È consigliabile, pertanto, proseguire il programma di sorveglianza nonostante l'eventuale sieroconversione indotta dalla terapia o l'assenza di progressione evidente di malattia [16].

Ulteriori fattori associati all'aumentato rischio di HCC sono l'elevata concentrazione sierica di AFP ( $>20 \mathrm{ng} / \mathrm{mL}$ ), la presenza di noduli epatici rigenerativi, di cellule displastiche e di rigenerazione irregolare (distribuzione disorganizzata della rigenerazione epatocitaria totalmente indipendente dall'architettura lobulare) alla biopsia epatica [24].

In presenza di tali fattori, gli autori delle linee guida AASLD consigliano di considerare l'utilità di un programma di sorveglianza sia pure al di fuori delle raccomandazioni sopra riportate [16].

\section{Test di sorveglianza}

Per definire la presenza di patologia occorre identificare indagini idonee per sensibilità e specificità; l'accuratezza diagnostica di ogni test correla con la prevalenza della patologia nella popolazione in studio.

- Il test sierologico utilizzato nella sorveglianza dell'HCC è il dosaggio dell'AFP, una glicoproteina prodotta durante lo sviluppo fetale nel sacco vitellino e nel fegato. Tale proteina è presente in concentrazioni molto basse nel sangue $(<10 \mathrm{ng} / \mathrm{mL})$ alla nascita e, nel corso della vita adulta, può però essere prodotta da cellule neoplastiche (tumori del fegato e del testicolo) con conseguenti maggiori 
Tabella 2 Test di sorveglianza per HCC.

\begin{tabular}{lll}
\hline & $\begin{array}{l}\text { Sensibilità } \\
(\%)\end{array}$ & $\begin{array}{l}\text { Specificità } \\
(\%)\end{array}$ \\
\hline $\begin{array}{l}\text { Alfa-fetoproteina } \\
\text { (cut-off: } 20 \mathrm{ng} / \mathrm{mL})\end{array}$ & 60 & 91 \\
normali $<10 \mathrm{ng} / \mathrm{mL}$ & & \\
\hline Ecografia epatica & $65-80$ & $>90$ \\
\hline
\end{tabular}

concentrazioni sieriche. Il cut-off è pari a $20 \mathrm{ng} / \mathrm{mL}$, in quanto migliore compromesso tra sensibilità e specificità, $60 \%$ e $91 \%$, rispettivamente (tabella 2) [25]. Benché l'AFP non sia un test ottimale, viene impiegato nella sorveglianza e nella diagnostica dell'HCC e assume un elevato valore predittivo positivo quando $>200 \mathrm{ng} / \mathrm{mL}$ in presenza di massa epatica su fegato cirrotico. Valori di AFP persistentemente elevati costituiscono un fattore di rischio per lo sviluppo di HCC [26].

- L'impiego della des-gamma-carbossiprotrombina (DCP), una forma alterata di protrombina prodotta da cellule neoplastiche e incapace di legare ioni calcio indispensabili per la sua attività, è stato valutato in numerosi studi; attualmente non emergono evidenze circa la sua utilità come test di sorveglianza [27].

- Gli ultrasuoni sono l'esame di sorveglianza strumentale attualmente più impiegato (sensibilità: $65-80 \%$, specificità $>90 \%$ ), con performance migliori di qualunque test sierologico (tabella 2) [28]. Si tratta però di una tecnica operatoredipendente e con minore sensibilità in soggetti obesi. Le linee guida della British Society of Gastroenterology consigliano l'utilità di formare operatori dedicati all'identificazione precoce dell'HCC in soggetti cirrotici (livello II) $[29,30]$.

- L'impiego combinato di AFP ed ecografia epatica aumenta i riscontri di HCC (livello II) [16], comportando però un incremento di costi e di falsi positivi. La non invasività di tali indagini favorisce una buona compliance da parte dei pazienti.

- Alcuni autori hanno proposto, come sorveglianza per HCC, la tomografia computerizzata trifasica: a oggi non vi sono studi che valutino l'utilità di questa tecnica nella diagnosi precoce e nel rapporto costo/beneficio [31].

\section{Timing}

- L'intervallo di sorveglianza è determinato dalla crescita tumorale, non dal rischio di HCC che caratterizza il singolo paziente.

- L'ideale intervallo di sorveglianza non è ancora stato definito.

- Sulla base del tempo di raddoppiamento delle cellule neoplastiche, l'osservazione andrebbe condotta ogni 612 mesi. Uno studio retrospettivo [32] non ha evidenziato differenze in termini di sopravvivenza tra pazienti indagati ogni 6 e 12 mesi e, secondo un recente RCT [33], non ne sussistono se la sorveglianza è condotta a 3 o 6 mesi.

- Uno studio svolto con l'impiego della tecnica ultrasonografica ha evidenziato che l'HCC è un tumore a crescita lenta: il tempo medio di raddoppiamento è stato stimato in
117 giorni (range: 29-398 giorni) e nella maggior parte dei casi è inferiore a 150 giorni [33].

- L'intervallo di sorveglianza di 6 mesi consentirebbe di identificare la maggior parte delle lesioni neoplastiche prima che raggiungano un diametro $>5 \mathrm{~cm}$.

\section{Diagnosi}

La diagnosi di HCC comprende metodiche sierologiche, citoistopatologiche e radiologiche le cui modalità di utilizzo sono ben individuate sia dalle linee guida EASL [21] sia dalle linee guida AASLD [16].

Le tecniche radiologiche associano all'elevata affidabilità diagnostica la possibilità di effettuare al contempo la corretta stadiazione del tumore.

L'ultrasonografia costituisce la metodica più utilizzata per lo screening e per la sorveglianza; minore è il suo ruolo nella diagnostica, con sensibilità e specificità non ben definite [34]. Nessuna delle caratteristiche ultrasonografiche delle lesioni focali epatiche è specifica per HCC.

La risonanza magnetica (RM) e la tomografia computerizzata (TC) con studio trifasico del fegato sono le metodiche più frequentemente impiegate per la diagnosi; la maggiore disponibilità di apparecchiature TC ha reso quest'ultima la più usata in assoluto. La vascolarizzazione dell'HCC è tipicamente fornita da rami dell'arteria epatica, mentre il rimanente fegato riceve sangue arterioso e soprattutto portale. Le caratteristiche dell'HCC durante lo studio trifasico sono una precoce presa di contrasto (arterial enhancement) con conseguente aspetto iperdenso e una successiva ipo-isodensità nella fase portale (washout). La presenza di precoce arterial enhancement e di successivo washout ha una sensibilità complessiva del $90 \%$ e una specificità del 95\%, con un lieve vantaggio a favore della $\mathrm{RM}$ rispetto alla TC. Le performance diagnostiche di queste due metodiche sono correlate alle dimensioni delle lesioni: in tumori $>2 \mathrm{~cm}$, la RM ha un'accuratezza diagnostica $>90 \%$, che si riduce al $33 \%$ per dimensioni $<2 \mathrm{~cm}$ [35]. Il $71 \%$ dei pazienti con HCC evidenzia le caratteristiche trifasiche tipiche a entrambe le metodiche.

Recenti studi basati sull'utilizzo dell'ecotomografia (ECT) con mezzo di contrasto hanno dimostrato come questa metodica possa affiancarsi alla TC e RM nella diagnosi non invasiva di HCC [36].

Valori di AFP $>200 \mathrm{ng} / \mathrm{mL}$ sono altamente specifici per la diagnosi di HCC in pazienti cirrotici con contemporaneo riscontro radiologico di lesione epatica focale [37]. Purtroppo la sensibilità dell'AFP è molto bassa per tale cutoff (22\%); complessivamente, solo un terzo dei pazienti con $\mathrm{HCC}$ ha livelli di AFP $>100 \mathrm{ng} / \mathrm{mL}$.

La sequenza delle metodiche da utilizzare per la diagnostica dipende dalle dimensioni della lesione (fig. 1).

\section{Lesioni di diametro superiore a $2 \mathrm{~cm}$}

Il riscontro ecografico di una lesione focale $>2 \mathrm{~cm}$ in un fegato cirrotico è altamente sospetto per HCC.

Se l'AFP è $>200 \mathrm{ng} / \mathrm{mL}$ o le caratteristiche radiologiche TC o RM sono quelle di una lesione unica $>2 \mathrm{~cm}$ o multifocale con precoce presa di contrasto e successivo washout portale, la biopsia non è essenziale e il sospetto è confermato $[16,37]$ (livello II). 


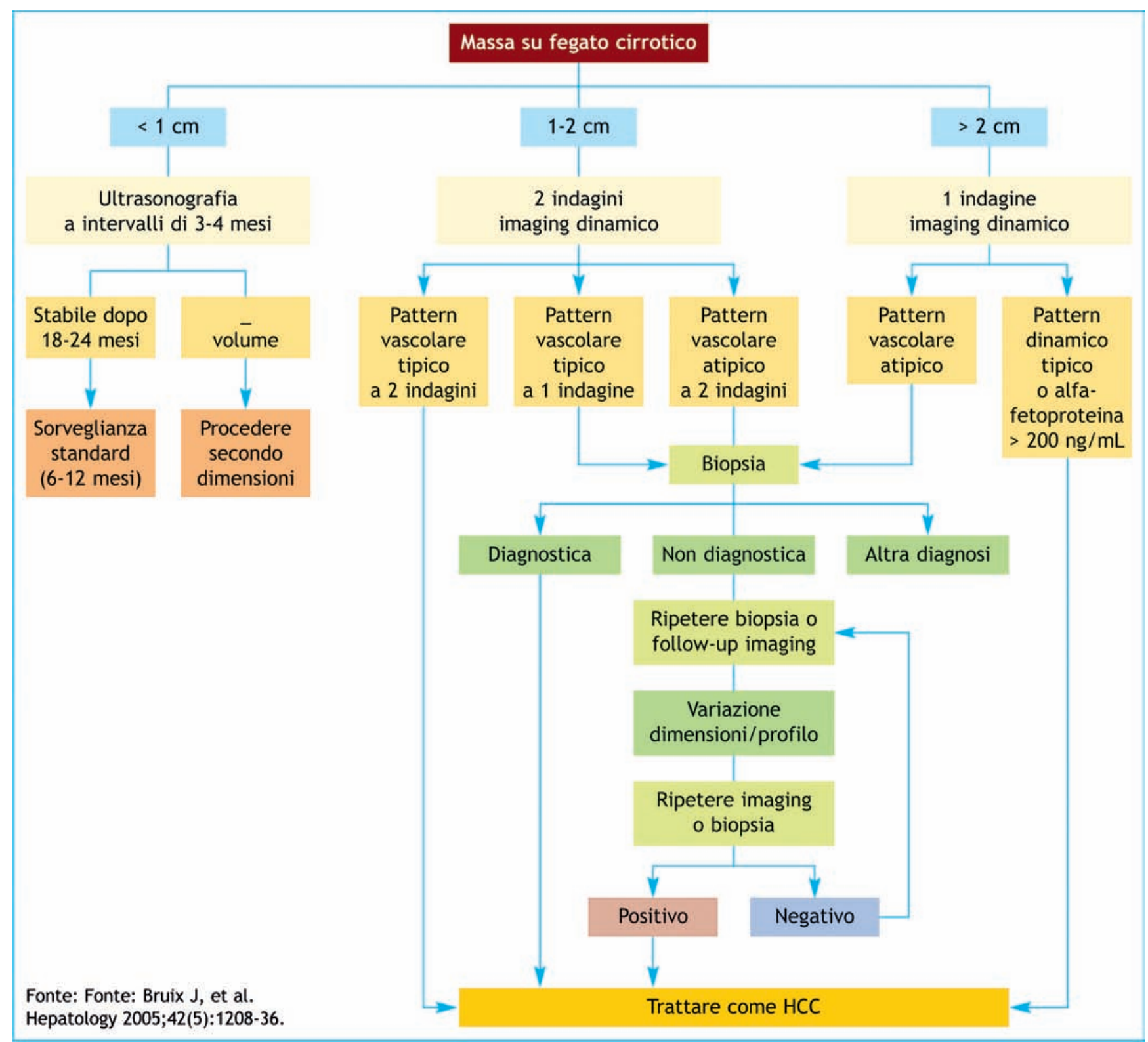

Figura 1 Algoritmo per il management di nodulo riscontrato all'ecotomografia in corso di sorveglianza per HCC.

Se le caratteristiche radiologiche, invece, sono atipiche, deve essere considerata la biopsia, analogamente a una lesione epatica focale in un fegato non cirrotico a prescindere dalle caratteristiche TC o RM (livello II).

\section{Lesioni di diametro uguale a $1-2 \mathrm{~cm}$}

Il riscontro, durante un programma di sorveglianza, di una lesione di diametro pari a $1-2 \mathrm{~cm}$ in un fegato cirrotico è sospetto per HCC.

Le linee guida EASL [21] raccomandano la biopsia a prescindere dal profilo vascolare della lesione, biopsia che può porre tanti più problemi quanto più la lesione ha dimensioni ridotte: falsi negativi da mancato campionamento, disaccordo fra i patologi nella linea di demarcazione fra displasia e $\mathrm{HCC}$ ben differenziato, difficoltà a distinguere un HCC ben differenziato dal tessuto normale. Il rischio di insemenzamento di cellule neoplastiche lungo il tragitto dell'ago da biopsia è considerato non comune, mentre il rischio di sanguinamento non differisce probabilmente da quello di un fegato cirrotico. Anche la caratterizzazione mediante le tecniche di immagine può essere difficile. Lesioni $<2 \mathrm{~cm}$ possono presentare il tipico arterial enhancement senza il venous washout nella fase portale; fino al $25 \%$ di queste lesioni rimangono stabili nel tempo o regrediscono. Le linee guida AASLD [16] consigliano la conferma del caratteristico pattern vascolare mediante l'impiego di due tecniche di immagine dinamica. Nel caso di assenza di tale conferma, anche a una sola metodica, viene consigliata la biopsia. Se la biopsia è negativa, viene raccomandato uno stretto follow-up mediante TC/RM (buona pratica a 3 mesi), ripetendo la biopsia se le dimensioni della lesione aumentano in assenza di pattern vascolare tipico. 


\section{Lesioni di diametro inferiore a $1 \mathrm{~cm}$}

Lesioni $<1 \mathrm{~cm}$ riscontrate in un fegato cirrotico durante un programma di sorveglianza hanno una bassa probabilità di essere HCC [38]. Tale probabilità si riduce ulteriormente se non presentano arterial enhancement alla valutazione di imaging dinamico. L'elevato rischio di progressione di queste lesioni verso un HCC consiglia di ridurre gli intervalli di sorveglianza ultrasonografica a 3-4 mesi [39].

\section{Stadiazione}

La prognosi dell'HCC varia in relazione allo stadio di malattia e al trattamento effettuato. Nel seguito si elencano alcuni dei principali fattori prognostici.

- Stadio del tumore: definito dal numero e dalla dimensione dei noduli, dalla presenza o assenza di invasione vascolare, dalla presenza o assenza di estensione extraepatica.

- Stato funzionale epatico: definito dalla classe Child-Pugh [40] e dalla presenza o assenza di ipertensione portale.

- Stato fisico generale, secondo la classificazione dello Eastern Cooperative Oncology Group (ECOG) [41].

- Presenza di sintomi di neoplasia.

L'eziologia non è stata identificata come fattore prognostico indipendente.

I sistemi di classificazione e stadiazione dovrebbero suddividere i pazienti in sottogruppi a outcome significativamente differente e contemporaneamente dovrebbero indirizzare la scelta terapeutica.

Le linee guida americane [16] ed europee [21] riconoscono come sistema di classificazione che soddisfa tali caratteristiche il Barcelona Clinic Liver Cancer (BCLC) [42] (tabella 3). La classificazione BCLC correla la stadiazione con la strategia terapeutica e individua gli standard terapeutici per ciascuno stadio di neoplasia: pazienti con HCC in stadio precoce candidabili a terapie curative, pazienti con HCC in stadio intermedio o avanzato che possono beneficiare di trattamenti palliativi e, infine, pazienti in stadio terminale con prognosi severa (fig. 2) [43].

\section{Stadi precoci: $0-\mathrm{A}$}

- Includono pazienti con funzionalità epatica conservata (Child-Pugh A-B) con HCC singolo $<5 \mathrm{~cm}$ o 3 noduli $\leq 3 \mathrm{~cm}$.

- Le possibilità di trattamento comprendono la resezione chirurgica, il trapianto di fegato e l'ablazione percutanea (trattamenti curativi).

- La sopravvivenza raggiunge il $50-70 \%$ a 5 anni dopo il trattamento curativo $[1,44]$.
Le variabili che influenzano la scelta del tipo di trattamento sono lo stadio del tumore e la funzionalità epatica.

- Lo stadio del tumore è definito dalla dimensione del nodulo principale e dalla multicentricità (singolo $\leq 2 \mathrm{~cm}$, $\mathrm{cm}$, singolo $2-5 \mathrm{~cm}, 3$ noduli $\leq 3 \mathrm{~cm}$ ), con outcome significativamente differenti per ciascuna categoria così identificata. Nella categoria del tumore $<2 \mathrm{~cm}$ è stato introdotto il concetto di HCC in stadio molto precoce o carcinoma in situ, lesione ben differenziata contenente dotti biliari e vene portali, senza invasione delle strutture. In tali pazienti la sopravvivenza a 5 anni dopo trattamento risulta particolarmente alta (resezione: 89\%; trattamento percutaneo: $71 \%$ ), mentre la ricorrenza di malattia è bassa ( $8 \%$ a 3 anni) [1].

- Le variabili correlate allo stato funzionale epatico sono importanti nei pazienti non candidati al trapianto; l'assenza di ipertensione portale e valori normali di bilirubina sono i principali predittori di sopravvivenza in caso di resezione di singolo nodulo. In modo analogo, la classe Child-Pugh A è la principale variabile prognostica in caso di ablazione percutanea, insieme alla dimensione del tumore e alla risposta al trattamento [45].

Poiché il trapianto di fegato può potenzialmente trattare sia la neoplasia sia l'insufficienza epatica, le variabili più strettamente legate all'HCC sono state identificate come fattori prognostici (singolo nodulo $\leq 5 \mathrm{~cm}$ o 3 noduli $\leq 3 \mathrm{~cm}$ ), secondo i criteri di Milano [46].

\section{Stadio intermedio: B}

- Include pazienti nelle classi Child-Pugh A e B con HCC voluminoso/multifocale senza sintomi di malattia né invasione vascolare o estensione extraepatica.

- Il trattamento standard è la chemioembolizzazione transarteriosa (TACE) [47].

- La sopravvivenza media in assenza di terapia è di 16 mesi; la TACE aumenta la sopravvivenza a 19-20 mesi.

\section{Stadio avanzato: C}

- Include pazienti che presentano sintomi di malattia o classe ECOG 1-2 o invasione vascolare/estensione extraepatica.

- Questi pazienti sono candidati all'arruolamento in trial terapeutici con nuovi agenti. Fino al 2006 non esisteva un trattamento approvato di prima linea. Recentemente sono stati pubblicati i risultati di uno studio che riporta un incremento della sopravvivenza rispetto al placebo in pazienti con HCC in stadio avanzato trattati con sorafenib, inibitore delle tirosinchinasi $[48,49]$.

Tabella 3 Classificazione-stadiazione clinica Barcelona Clinic Liver Cancer (BCLC).

\begin{tabular}{llllll}
\hline & $\begin{array}{l}\text { Stadio molto } \\
\text { precoce (0) }\end{array}$ & $\begin{array}{l}\text { Stadio } \\
\text { precoce (A) }\end{array}$ & $\begin{array}{l}\text { Stadio } \\
\text { intermedio (B) }\end{array}$ & $\begin{array}{l}\text { Stadio } \\
\text { avanzato (C) }\end{array}$ & $\begin{array}{l}\text { Stadio } \\
\text { terminale (D) }\end{array}$ \\
\hline Child-Pugh & A & A-B & A-B & A-B & C \\
\hline Performance status & 0 & 0 & 0 & $1-2$ & $3-4$ \\
\hline Stadio del tumore & $\begin{array}{l}1 \mathrm{HCC}<2 \mathrm{~cm} \\
\text { Carcinoma in situ }\end{array}$ & $\begin{array}{l}1 \mathrm{HCC}<5 \mathrm{~cm} \text { o } \\
3 \text { noduli } \leq 3 \mathrm{~cm}\end{array}$ & Multinodulare & $\begin{array}{l}\text { Invasione } \\
\text { portale o N1/M1 }\end{array}$ & $\begin{array}{l}\text { Stadio } \\
\text { terminale }\end{array}$ \\
\hline
\end{tabular}

Fonte: Llovet JM, et al. Semin Liver Dis 1999;19(3):329-38. 


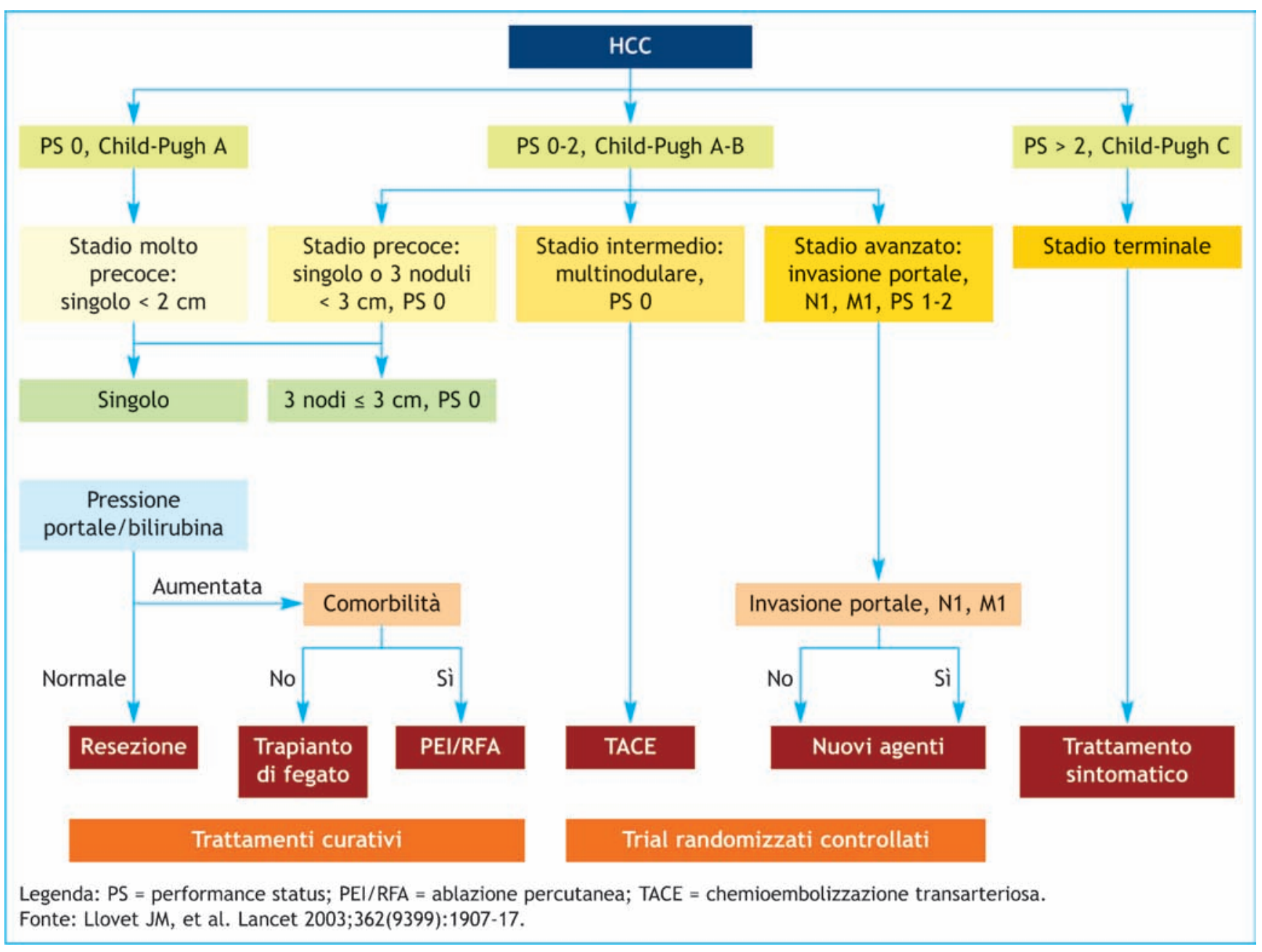

Figura 2 Classificazione-stadiazione Barcelona Clinic Liver Cancer (BCLC).

- La sopravvivenza media a 1 anno in assenza terapia è pari a $50 \%$.

\section{Stadio terminale: $\mathbf{D}$}

- Include pazienti con neoplasia estesa che determina un importante deterioramento del performance status (ECOG 3-4) e/o una severa compromissione della funzionalità epatica (Child-Pugh C).

- La sopravvivenza media è pari a 3-4 mesi.

\section{Trattamento}

- Le strategie terapeutiche che offrono un'alta probabilità di risposta completa, potenzialmente curative, comprendono la resezione chirurgica, il trapianto di fegato e l'ablazione percutanea [1].

- Tra le terapie non curative, l'unica che ha dimostrato di migliorare significativamente la sopravvivenza è risultata la TACE [47], mentre tale miglioramento non è significativo con l'utilizzo di sorafenib [48,49]. L'embolizzazione arteriosa senza chemioterapia e la brachiterapia hanno dimostrato attività antitumorale, senza prove della loro efficacia in termini di sopravvivenza.

\section{Resezione chirurgica}

- La resezione chirurgica è il trattamento di scelta nei pazienti non cirrotici ( $5 \%$ dei pazienti nei Paesi occidentali, $40 \%$ in Asia); in soggetti con funzionalità epatica preservata possono essere effettuate resezioni maggiori con basso rischio di complicanze e sopravvivenza a 5 anni $>50 \%$ [50]. La selezione dei candidati si basa sulla definizione della funzionalità epatica e dell'estensione del tumore. I principali fattori predittivi di sopravvivenza e ricorrenza sono riportati nelle tabelle 4 e 5 [19]. In Europa e negli Stati Uniti, la selezione dei candidati si basa sulla valutazione della presenza di ipertensione portale, identificata mediante cateterismo delle vene sovraepatiche o attraverso segni clinici (una conta piastrinica $<100.000 / \mathrm{mm}^{3}$ associata a splenomegalia costituisce indice di ipertensione portale). La misurazione della pressione portale mediante cateterismo ha un ruolo centrale; l'ipertensione portale può essere definita severa, senza necessità di cateterismo, in presenza di varici esofagee o ascite in trattamento diuretico. Normali valori di bilirubina e assenza di ipertensione portale (pressione venosa epatica $<10 \mathrm{mmHg}$ ) sono i migliori predittori di un ottimo outcome postchirurgico, associandosi a un rischio di insufficienza epatica postintervento quasi nullo [19]. 
Tabella 4 Fattori predittivi di sopravvivenza postresezione chirurgica.

\begin{tabular}{|c|c|c|}
\hline Autore & N. pazienti & Variabili \\
\hline Llovet & 77 & $\begin{array}{l}\text { Ipertensione portale }( \pm) \\
\text { Bilirubina }(<1 \mathrm{mg} / \mathrm{dL},>1 \mathrm{mg} / \mathrm{dL})\end{array}$ \\
\hline Zhao & 1.000 & $\begin{array}{l}\text { Cirrosi }( \pm) \\
\text { Gamma-GT } \\
\text { Numero di noduli (singolo vs multipli) } \\
\text { Invasione portale }( \pm)\end{array}$ \\
\hline Vauthey & 557 & $\begin{array}{l}\text { Invasione vascolare micro e macroscopica }( \pm \text { ) } \\
\text { Numero di noduli (singolo vs multipli) } \\
\text { Dimensioni }(<5 \mathrm{~cm},>5 \mathrm{~cm} \text { ) } \\
\text { Fibrosi (score di Ishak } 0-4 \text { vs } 5-6 \text { ) }\end{array}$ \\
\hline Poon & 518 & $\begin{array}{l}\text { Invasione vascolare maggiore } \\
\text { Invasione microvascolare } \\
\text { Dimensioni }>5 \mathrm{~cm} \\
\text { Noduli multipli/malattia bilobare } \\
\text { Cirrosi } \\
\text { AST }>50 \mathrm{UI} / \mathrm{L} \\
\text { Invasione di organi adiacenti }\end{array}$ \\
\hline Ikai & 12.118 & $\begin{array}{l}\text { Dimensione }(\leq 2 \mathrm{~cm}, 2-5 \mathrm{~cm}, 5-10 \mathrm{~cm},>10 \mathrm{~cm}) \\
\text { Numero di noduli (singolo vs multipli) } \\
\text { Alfa-fetoproteina }(<20 \mathrm{ng} / \mathrm{mL}, 20-200 \mathrm{ng} / \mathrm{mL}, 200-1.000 \mathrm{ng} / \mathrm{mL},>1.000 \mathrm{ng} / \mathrm{mL} \text { ) } \\
\text { Grado del danno epatico }(\mathrm{A}, \mathrm{B}, \mathrm{C}) \\
\text { Invasione vascolare }( \pm) \\
\text { Curabilità chirurgica }( \pm)\end{array}$ \\
\hline
\end{tabular}

Fonte: Llovet JM, et al. J Hepatol 2008;48(Suppl 1):S20-37.

Tabella 5 Fattori predittivi di ricorrenza postresezione chirurgica.

\begin{tabular}{|c|c|c|}
\hline Autore & N. pazienti & Variabili \\
\hline Belghiti & 47 & $\begin{array}{l}\text { Dimensioni del nodulo } \\
\text { Alfa-fetoproteina }\end{array}$ \\
\hline Kumada & 57 & $\begin{array}{l}\text { Alfa-fetoproteina } \\
\text { HCC multinodulare } \\
\text { Dimensioni del nodulo }\end{array}$ \\
\hline Llovet & 77 & $\begin{array}{l}\text { Grado di } \\
\text { differenziazione } \\
\text { Multinodularità } \\
\text { Noduli satelliti }\end{array}$ \\
\hline $\begin{array}{l}\text { Imamura } \\
\text { - Ricorrenza precoce } \\
\text { ( }<2 \text { anni) } \\
\text { - Ricorrenza tardiva } \\
\text { ( }>2 \text { anni) }\end{array}$ & $\begin{array}{l}123 \\
61\end{array}$ & $\begin{array}{l}\text { Invasione vascolare } \\
\text { Alfa-fetoproteina } \\
\text { Resezione non } \\
\text { anatomica } \\
\text { Multinodularità } \\
\text { Attività virale } \\
\text { Classificazione HCC }\end{array}$ \\
\hline Ercolani & 224 & $\begin{array}{l}\text { Numero di noduli } \\
\text { AST }\end{array}$ \\
\hline
\end{tabular}

Fonte: Llovet JM, et al. J Hepatol 2008;48(Suppl 1):S20-37.
L'estensione del tumore può essere stimata mediante TC o RM con mezzo di contrasto. Tuttavia, anche le tecniche più innovative sottostimano la stadiazione in più del $30 \%$ dei casi. L'ecografia intraoperatoria è in grado di rilevare i noduli di dimensioni pari a $0,5-1 \mathrm{~cm}$ ed è considerata lo standard per escludere la presenza di lesioni satellite e per guidare l'intervento. Le variabili associate in modo significativo con la prognosi sono le dimensioni, il numero e la presenza di invasione vascolare. La dimensione del tumore tuttavia non rappresenta un definito fattore limitante l'intervento. Il Japanese Nationwide Survey [51] ha evidenziato che la dimensione $<2 \mathrm{~cm}$ è un predittore indipendente di sopravvivenza con tassi di ricorrenza a 5 anni inferiori al $66 \%$ per gli $\mathrm{HCC} \leq 2 \mathrm{~cm}$ e pari a $37 \%$ per lesioni $>5 \mathrm{~cm}$. Nello stesso studio [51], la sopravvivenza a 5 anni era del $57 \%$ per i tumori singoli, riducendosi al $26 \%$ in presenza di 3 o più noduli.

- La resezione nei pazienti con cirrosi richiede un'attenta selezione dei candidati (precisa conoscenza dello stadio di malattia, attenta valutazione della morbilità/mortalità postoperatoria, adeguata esperienza dell'équipe); negli ultimi anni, con il miglioramento delle tecniche chirurgiche e del management perioperatorio, la mortalità perioperatoria in questo setting è scesa al di sotto del $3 \%$, con sopravvivenza a 5 anni del $50 \%$.

- Trattamento adiuvante: la recidiva di malattia dopo resezione a 5 anni raggiunge il $70 \%$, considerando sia le recidive dovute a disseminazione (metastasi intraepatiche) sia le neoplasie de novo [16]: il $60-70 \%$ delle recidive corrisponde a metastasi intraepatiche non riconosciute al 
momento della resezione, mentre nel $30-40 \%$ dei casi si tratta di HCC de novo. I più importanti predittori di ricorrenza sono la presenza di invasione microvascolare e/o siti addizionali di neoplasia diversi dalla lesione primaria; la recidiva conseguente a disseminazione generalmente si sviluppa nei primi 3 anni di follow-up.

Attualmente non vi sono strategie terapeutiche in grado di ridurre efficacemente il rischio di recidiva (fig. 3) [19]. La chemioembolizzazione neoadiuvante e/o la chemioterapia adiuvante non hanno evidenziato effetti in termini di prevenzione di recidiva [11,21]. L'irradiazione interna con I131 e lipiodol ha dimostrato effetto positivo in un unico RCT [52]; l'effetto osservato merita ulteriori approfondimenti. L'immunoterapia con linfociti attivati si è rivelato in grado di ridurre la prima recidiva in un trial su 150 pazienti [53]. Un effetto sovrapponibile è stato osservato con i retinoidi nella prevenzione delle ricorrenze de novo [54]. L'interferone ha prodotto effetti positivi in alcuni RCT, compreso uno studio condotto in pazienti dopo resezione/ablazione percutanea [19]; recentemente l'interferone a basso dosaggio per più di 6 anni, in uno studio caso-controllo svolto su pazienti sottoposti a iniziale termoablazione con radiofrequenza (RFA) curativa, ha determinato un significativo aumento della sopravvivenza e una riduzione della ricorrenza di HCC [55].

Nessuna delle opzioni terapeutiche descritte è suffragata da livelli di evidenza tali da consigliarne l'utilizzo nella pratica clinica.

Il trattamento delle recidive non è attualmente standardizzato. La recidiva singola potrebbe beneficiare di una nuova resezione, ma nella maggior parte dei pazienti, dopo la resezione iniziale, le recidive sono multifocali e conseguenti alla disseminazione intraepatica del tumore primitivo.

\section{Recidiva reale $(50-70 \%)$}

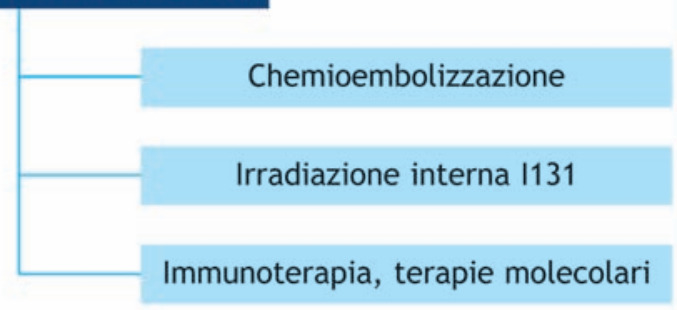

HCC de novo $(30-50 \%)$

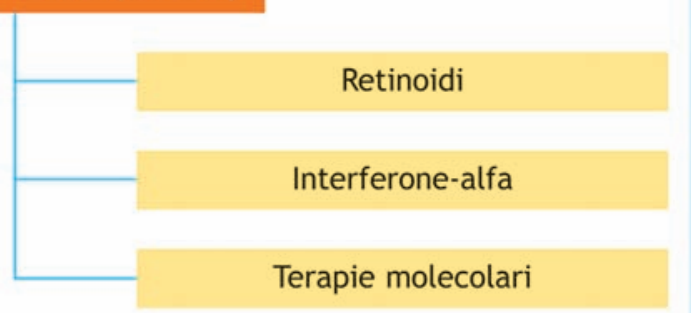

Fonte: modificato da Llovet JM, et al.

J Hepatol 2008;48(Suppl 1):S20-37.

Figura 3 Strategie di prevenzione delle recidive di HCC.

\section{Trapianto di fegato}

Secondo i criteri di Milano [46], il trapianto di fegato è il trattamento di scelta nei pazienti con HCC singolo di dimensioni $\leq 5 \mathrm{~cm}$ o con un numero massimo di 3 noduli $\leq 3 \mathrm{~cm}$.

In accordo con i suddetti criteri, le indicazioni al trapianto sono:

- HCC non resecabile in cirrosi epatica di qualunque eziologia;

- HCC non resecabile in assenza di malattia di fegato;

- HCC in cirrosi epatica e ridotta funzione parenchimale. Le controindicazioni assolute al trapianto sono:

- invasione macroscopica di vena porta e vene sovraepatiche;

- malattia extraepatica.

La sopravvivenza a 5 anni è $>70 \%$, con recidiva $<15 \%$.

Il principale problema del trapianto è costituito dall'esiguo numero di organi disponibili; l'allungamento dei tempi di attesa porta il $20 \%$ dei candidati ad abbandonare le liste per progressione di malattia. Per tali ragioni si adottano strategie terapeutiche neoadiuvanti: ablazione percutanea (PEI/RFA) o TACE [56].

\section{Ablazione percutanea}

L'ablazione percutanea è il trattamento di prima scelta in pazienti con HCC in stadio precoce non candidati alla resezione chirurgica né al trapianto [45].

Nei casi migliori, con l'ablazione si raggiunge una sopravvivenza a 5 anni del $40-70 \%$ [16,21]. Il miglior outcome si ottiene nei pazienti in classe $A$ di Child-Pugh con singola lesione di diametro $<3 \mathrm{~cm}$ [45]. I predittori indipendenti di sopravvivenza sono la risposta completa iniziale, la classe Child-Pugh, il numero e la dimensione dei noduli, i livelli basali di AFP.

La distruzione delle cellule tumorali si può ottenere con l'iniezione di sostanze chimiche (etanolo, acido acetico) o attraverso la modificazione della temperatura (radiofrequenze, microonde, laser, crioterapia). Negli ultimi anni sono stati pubblicati almeno 6 RCT (totale 822 pazienti): quattro di questi studi hanno paragonato l'iniezione percutanea con acido acetico (PEI) con l'ablazione mediante radiofrequenze (RFA/RITA) [16]. L'efficacia della RFA in lesioni $<2 \mathrm{~cm}$ è sovrapponibile a quella della PEI, con il vantaggio di un numero inferiore di sedute; la RFA si dimostra significativamente più efficace in lesioni di diametro $>2 \mathrm{~cm}[16,21]$.

L'efficacia della terapia ablativa è valutata mediante TC trifasica a un mese dalla procedura [21].

\section{Chemioembolizzazione}

L'embolizzazione arteriosa è la strategia terapeutica maggiormente utilizzata in caso di lesioni non curabili $[1,16,21$, 47,48].

I candidati ideali sono i pazienti con funzione epatica conservata e lesioni multinodulari asintomatiche senza invasione vascolare o estensione extraepatica, mentre deve essere evitata in caso di insufficienza epatica (Child-Pugh B-C), poiché il danno ischemico può determinarne un peggioramento.

L'embolizzazione arteriosa selettiva induce una necrosi ischemica a carico del tessuto neoplastico tributario 
altamente vascolarizzato. L'embolizzazione può essere effettuata durante angiografia e viene definita "embolizzazione transarteriosa" (TAE); quando la TAE viene associata all'iniezione in arteria epatica di agenti chemioterapici, la procedura assume il nome di "chemioembolizzazione transarteriosa" (TACE). I chemioterapici utilizzati sono: doxorubicina, adriamicina, mitomicina e cisplatino.

La TACE ottiene una risposta parziale nel $15-55 \%$ dei pazienti $[1,16,47]$ e ritarda in modo significativo la progressione e l'invasione vascolare, con un miglioramento della sopravvivenza media [57].

La risposta al trattamento viene stabilita in base alla riduzione dell'AFP, all'identificazione dell'area di necrosi intratumorale e alla riduzione delle dimensioni alla TC trifasica o alla RM [21]. Le indagini dinamiche (TC o RM) vengono effettuate 30-60 giorni dopo il trattamento, proseguendo poi nel follow-up con ECT dopo ulteriori 3 mesi, TC trifasica a 6 mesi e così via.

\section{Altre terapie non curative}

- Le terapie loco-regionali diverse dalla TACE non hanno finora dimostrato vantaggi in termini di sopravvivenza. Alcune procedure, come la brachiterapia con I131 associato a lipiodol o la lipiodolizzazione arteriosa, inducono una risposta nel $20 \%$ dei casi; in particolare, la brachiterapia sta acquisendo un ruolo come alternativa alla TACE in caso di HCC in stadio intermedio-avanzato. Uno studio di coorte [58] ha dimostrato che il trattamento con Y-90 ottiene una buon tasso di risposta e prolunga la sopravvivenza nell'HCC avanzato a 12 mesi.

- La terapia ormonale con tamoxifene e la radioterapia esterna non hanno dimostrato effetto positivo sulla sopravvivenza; tali trattamenti sono da evitare nel caso di HCC avanzato [16,21].

- La chemioterapia sistemica è stata valutata in 9 RCT [16,21]: gli agenti più attivi sono la doxorubicina (tasso di risposta del $10-15 \%)$ e il cisplatino. Non vi sono effetti positivi in termini di sopravvivenza.

- Gli iniziali risultati molto incoraggianti ottenuti con l'interferone e l'octreotide non sono stati confermati da successivi studi; 3 studi hanno evidenziato effetti negativi in caso di HCC avanzato [59].

- Terapia molecolare: molti farmaci in studio sono bloccanti dei recettori di membrana tirosinchinasici (TKR), i cui ligandi comprendono EGF (Endothelial Growth Factor), PDGF (Platelet-Derived Growth Factor), VEGF (Vascular Endothelial Growth Factor) e HGF (Hepatocyte Growth Factor). Questi ligandi attivano la via di trasmissione del segnale Ras/MAPK, inducendo la trascrizione di geni della famiglia AP1, come c-fos e c-jun, elementi essenziali nel controllo della proliferazione cellulare [16,21,48,49].

- Sorafenib è un inibitore multichinasico, recentemente approvato dalla Food and Drug Administration (FDA) e dall'Agenzia Europea per i Medicinali (EMEA) per il trattamento dell'HCC, la cui azione si esplica mediante l'inibizione dell'angiogenesi e della proliferazione cellulare mediante blocco del segnale Ras/MAPK. Sorafenib aumenta la sopravvivenza libera da malattia nel carcinoma renale [60]; recentemente è stato pubblicato uno studio [49] in cui è emerso che, nei pazienti con HCC avanzato non precedentemente trattati, la terapia con sorafenib (400 mg 2 volte al giorno, riducibili a $400 \mathrm{mg}$ in unica somministrazione o $400 \mathrm{mg}$ ogni 2 giorni in caso di effetti collaterali) aumenta la sopravvivenza (endpoint primario) di 3 mesi rispetto al placebo e ritarda la progressione radiologica (endpoint secondario).

- Un blocco efficace del segnale Ras/MAPK si può ottenere con l'utilizzo di anticorpi monoclonali contro EGFR (cetuximab) o ErbB2/Her2/neu (trastuzumab); in alternativa l'inibizione può essere raggiunta con piccole molecole dirette contro il domain catalitico di EGFR, come erlotinib o gefitinib. Lapatinib blocca invece contemporaneamente EGFR e Her2. Tutti questi farmaci sono attualmente in fase II di studio [11,21].

L'HCC è un tumore riccamente vascolarizzato e in precedenza è stata riportata un'aumentata espressione di fattori proangiogenetici come VEGF, PDGF e angiopoietina 2; da queste premesse si comprende il razionale dell'utilizzo di terapie antiangiogeniche nell'HCC, consistenti in anticorpi monoclonali quali bevacizumab o piccole molecole quali sunitinib e sorafenib [61]. La maggior parte di queste molecole sono al momento in fase II di studio.

\section{Conflitto di interessi}

Gli autori dichiarano di essere esenti da conflitto di interessi.

\section{Bibliografia}

[1] Hepatocellular Carcinoma: screening, diagnosis, and management. Natcher Conference Center, NIH; Bethesda, MD; April 1-3, 2004.

[2] Parkin DM, Bray F, Ferlay J, Pisani P. Global cancer statistics, 2002. CA Cancer J Clin 2005;55(2):74-108.

[3] Sangiovanni A, Del Ninno E, Fasani P, De Fazio C, Ronchi G, Romeo R, et al. Increased survival of cirrhotic patients with a hepatocellular carcinoma detected during surveillance. Gastroenterology 2004;126(4):1005-14.

[4] El-Serag HB, Mason AC. Rising incidence of hepatocellular carcinoma in the United States. N Engl J Med 1999;340(10):745-50.

[5] McGlynn KA, Tsao L, Hsing AW, Devesa SS, Fraumeni Jr JF. International trends and patterns of primary liver cancer. Int J Cancer 2001;94(2):290-6.

[6] World Health Organization. Mortality Database. WHO Statistical Information System. http://www.who.int/whosis.

[7] El-Serag HB. Hepatocellular carcinoma: an epidemiologic view. $\mathrm{J}$ Clin Gastroenterol 2002;35(5 Suppl 2):S72-8.

[8] Wands J. Hepatocellular carcinoma and sex. N Engl J Med 2007; 357(19):1974-6.

[9] El-Serag HB, Siegel AB, Davila JA, Shaib YH, Cayton-Woody M, McBride R, et al. Treatment and outcomes of treating of hepatocellular carcinoma among Medicare recipients in the United States: a population-based study. J Hepatol 2006;44(1):158-66.

[10] Capocaccia R, Sant M, Berrino F, Simonetti A, Santi V, Trevisani F, EUROCARE Working Group. Hepatocellular carcinoma: trends of incidence and survival in Europe and the United States at the end of the $20^{\text {th }}$ century. Am J Gastroenterol 2007;102(8): 1661-70.

[11] Yang HI, Lu SN, Liaw YF, et al., Taiwan Community-Based Cancer Screening Project Group. Hepatitis B e antigen and the risk of hepatocellular carcinoma. N Engl J Med 2002;347(3):168-74.

[12] Fattovich G, Stroffolini T, Zagni I, Donato F. Hepatocellular carcinoma in cirrhosis: incidence and risk factors. Gastroenterology 2004;127(5 Suppl 1):S35-50. 
[13] Bagnardi V, Blangiardo M, La Vecchia C, Corrao G. A metaanalysis of alcohol drinking and cancer risk. $\mathrm{Br} \mathrm{J}$ Cancer 2001;85(11):1700-5.

[14] Chen ZM, Liu BQ, Boreham J, Wu YP, Chen JS, Peto R. Smoking and liver cancer in China: case-control comparison of 36,000 liver cancer deaths vs. 17,000 cirrhosis deaths. Int J Cancer 2003;107(1):106-12.

[15] Elmberg M, Hultcrantz R, Ekbom A, Brandt L, Olsson S, Olsson R, et al. Cancer risk in patients with hereditary hemochromatosis and in their first-degree relatives. Gastroenterology 2003;125(6): 1733-41.

[16] Bruix J, Sherman M. Practice Guidelines Committee. American Association for the Study of Liver Diseases. Management of hepatocellular carcinoma. Hepatology 2005;42(5):120836.

[17] Zhang BH, Yang BH, Tang ZY. Randomized controlled trial of screening for hepatocellular carcinoma. J Cancer Res Clin Oncol 2004;130(7):417-22.

[18] Sarasin FP, Giostra E, Hadengue A. Cost-effectiveness of screening for detection of small hepatocellular carcinoma in Western patients with Child-Pugh class A cirrhosis. Am J Med 1996;101(4): 422-34.

[19] Llovet JM, Bruix J. Novel advancements in the management of hepatocellular carcinoma in 2008. J Hepatol 2008;48(Suppl 1): S20-37.

[20] Degos F, Christidis C, Ganne-Carrie N, Farmachidi J, Degott C, Guettier $C$, et al. Hepatitis $C$ virus related cirrhosis: time to occurrence of hepatocellular carcinoma and death. Gut 2000; 47(1):131-6.

[21] Bruix J, Sherman M, Llovet JM, Beaugrand M, Lencioni R, Burroughs AK, et al., EASL Panel of Experts on HCC. Clinical management of hepatocellular carcinoma. Conclusions of the Barcelona-2000 EASL conference. European Association for the Study of the Liver. J Hepatol 2001;35(3):421-30.

[22] Shaheen AA, Wan AF, Myers RP. FibroTest and FibroScan for the prediction of hepatitis C-related fibrosis: a systematic review of diagnostic test accuracy. Am J Gastroenterol 2007;102(11): 2589-600.

[23] Giordano TP, Kramer JR, Souchek J, Richardson P, El-Serag HB. Cirrhosis and hepatocellular carcinoma in HIV-infected veterans with and without the hepatitis C virus: a cohort study, 19922001. Arch Intern Med 2004;164(21):2349-54.

[24] Shibata M, Morizane T, Uchida T, Yamagami T, Onozuka Y, Nakano $M$, et al. Irregular regeneration of hepatocytes and risk of hepatocellular carcinoma in chronic hepatitis and cirrhosis with hepatitis-C-virus infection. Lancet 1998;351(9118):1773-7.

[25] Oka H, Tamori A, Kuroki T, Kobayashi K, Yamamoto S. Prospective study of alpha-fetoprotein in cirrhotic patients monitored for development of hepatocellular carcinoma. Hepatology 1994;19(1):61-6.

[26] Daniele B, Bencivenga A, Megna AS, Tinessa V. Alpha-fetoprotein and ultrasonography screening for hepatocellular carcinoma. Gastroenterology 2004;127(5 Suppl 1):S108-12.

[27] Koike Y, Shiratori Y, Sato S, Obi S, Teratani T, Imamura M, et al. Des-gamma-carboxy prothrombin as a useful predisposing factor for the development of portal venous invasion in patients with hepatocellular carcinoma: a prospective analysis of 227 patients. Cancer 2001;91(3):561-9.

[28] Bolondi L, Sofia S, Siringo S, Gaiani S, Casali A, Zironi Z, et al. Surveillance programme of cirrhotic patients for early diagnosis and treatment of hepatocellular carcinoma: a cost effectiveness analysis. Gut 2001;48(2):251-9.

[29] Ryder SD. British Society of Gastroenterology. Guidelines for the diagnosis and treatment of hepatocellular carcinoma (HCC) in adults. Gut 2003;52(Suppl 3):iii1-8.

[30] Colombo M, de Franchis R, Del Ninno E, et al. Hepatocellular carcinoma in Italian patients with cirrhosis. N Engl J Med 1991;325(10):675-80.
[31] Okuda K. Early recognition of hepatocellular carcinoma. Hepatology 1986;6(4):729-38.

[32] Trevisani F, De NS, Rapaccini G, et al., Italian Liver Cancer Group. Semiannual and annual surveillance of cirrhotic patients for hepatocellular carcinoma: effects on cancer stage and patient survival (Italian experience). Am J Gastroenterol 2002;97(3):734-44.

[33] Trinchet J, Beaugrand M. A randomized trial comparing 3 month vs. 6 month screening for HCC by ultrasonography in cirrhosis. ILCA's First Annual Conference Book of Abstracts, 2007: p. 11.

[34] El-Serag HB, Marrero JA, Rudolph L, Reddy KR. Diagnosis and treatment of hepatocellular carcinoma. Gastroenterology 2008;134(6):1752-63.

[35] Taouli B, Losada M, Holland A, Krinsky G. Magnetic resonance imaging of hepatocellular carcinoma. Gastroenterology 2004;127(5 Suppl 1):S144-52.

[36] Gaiani S, Celli N, Piscaglia F, Cecilioni L, Losinno F, Grangregorio $\mathrm{F}$, et al. Usefulness of contrast-enhanced perfusional sonography in the assessment of hepatocellular carcinoma hypervascular at spiral computed tomography. J Hepatol 2004;41(3): 421-6.

[37] Sherman M. Alphafetoprotein: an obituary. J Hepatol 2001;34(4): $603-5$.

[38] Kojiro M. Focus on dysplastic nodules and early hepatocellular carcinoma: an Eastern point of view. Liver Transpl 2004;10(2 Suppl 1):S3-8.

[39] Llovet JM, Di Bisceglie AM, Bruix J, et al. Panel of Experts in HCCDesign Clinical Trials. Design and endpoints of clinical trials in hepatocellular carcinoma. J Natl Cancer Inst 2008;100(10): 698-711.

[40] Pugh RN, Murray-Lyon IM, Dawson JL, Pietroni MC, Williams R. Transection of the oesophagus for bleeding oesophageal varices. Br J Surg 1973;60(8):646-9.

[41] Sørensen JB, Klee M, Palshof T, Hansen HH. Performance status assessment in cancer patients. An inter-observer variability study. Br J Cancer 1993;67(4):773-5.

[42] Llovet JM, Brú C, Bruix J. Prognosis of hepatocellular carcinoma: the BCLC staging classification. Semin Liver Dis 1999;19(3): 329-38.

[43] Llovet JM, Burroughs A, Bruix J. Hepatocellular carcinoma. Lancet 2003;362(9399):1907-17.

[44] Llovet JM, Schwartz M, Mazzaferro V. Resection and liver transplantation for hepatocellular carcinoma. Semin Liver Dis 2005; 25(2):181-200.

[45] Sala M, Llovet JM, Vilana R, et al., Barcelona Clínic Liver Cancer Group. Initial response to percutaneous ablation predicts survival in patients with hepatocellular carcinoma. Hepatology 2004;40(6):1352-60.

[46] Mazzaferro V, Regalia E, Doci R, et al. Liver transplantation for the treatment of small hepatocellular carcinomas in patients with cirrhosis. N Engl J Med 1996;334(11):693-9.

[47] Llovet JM, Bruix J. Systematic review of randomized trials for unresectable hepatocellular carcinoma: chemoembolization improves survival. Hepatology 2003;37(2):429-42.

[48] Llovet J, Ricci S, Mazzaferro V, Hilgard P, Raoul J, Zeuzem S, et al. Sorafenib improves survival in advanced hepatocellular carcinoma $(\mathrm{HCC})$ : results of a phase III randomized placebocontrolled trial (SHARP trial). 2007 ASCO Annual Meeting. J Clin Oncol 2007; 25:18S (Abstr LBA1).

[49] Llovet JM, Ricci S, Mazzaferro V, Hilgard P, Gane E, Blanc JF, et al., SHARP Investigators Study Group. Sorafenib in advanced hepatocellular carcinoma. N Engl J Med 2008;359(4):378-90.

[50] Belghiti J, Hiramatsu K, Benoist S, Massault P, Sauvanet A, Farges 0 . Seven hundred forty-seven hepatectomies in the 1990s: an update to evaluate the actual risk of liver resection. J Am Coll Surg 2000;191(1):38-46.

[51] Ikai I, Arii S, Kojiro M, Ichida T, Makuuchi M, Matsuyama Y, et al. Reevaluation of prognostic factors for survival after 
liver resection in patients with hepatocellular carcinoma in a Japanese nationwide survey. Cancer 2004;101(4):796802.

[52] Lau WY, Leung TW, Ho SK, Chan M, Machin D, Lau J, et al. Adjuvant intra-arterial iodine-131-labelled lipiodol for resectable hepatocellular carcinoma: a prospective randomised trial. Lancet 1999;353(9155):797-801.

[53] Takayama T, Sekine T, Makuuchi M, Yamasaki S, Kosuge T, Yamamoto J, et al. Adoptive immunotherapy to lower postsurgical recurrence rates of hepatocellular carcinoma: a randomised trial. Lancet 2000;356(9232):802-7.

[54] Muto Y, Moriwaki H, Ninomiya M, et al., Hepatoma Prevention Study Group. Prevention of second primary tumors by an acyclic retinoid, polyprenoic acid, in patients with hepatocellular carcinoma. N Engl J Med 1996;334(24):1561-7.

[55] Kudo M, Sakaguchi Y, Chung H, Hatanaka K, Hagiwara S, Ishikawa $\mathrm{E}$, et al. Long-term interferon maintenance therapy improves survival in patients with HCV-related hepatocellular carcinoma after curative radiofrequency ablation. A matched case-control study. Oncology 2007;72(Suppl 1):132-8.
[56] Lu DS, Yu NC, Raman SS, et al. Percutaneous radiofrequency ablation of hepatocellular carcinoma as a bridge to liver transplantation. Hepatology 2005;41(5):1130-7.

[57] Bruix J, Sala M, Llovet JM. Chemoembolization for hepatocellular carcinoma. Gastroenterology 2004;127(5 Suppl 1):S179-88.

[58] Kulik LM, Carr BI, Mulcahy MF, Lewandowski RJ, Atassi B, Ryu RK, et al. Safety and efficacy of $90 \mathrm{Y}$ radiotherapy for hepatocellular carcinoma with and without portal vein thrombosis. Hepatology 2008;47(1):71-81.

[59] Yuen MF, Poon RT, Lai CL, et al. A randomized placebo-controlled study of long-acting octreotide for the treatment of advanced hepatocellular carcinoma. Hepatology 2002;36(3):687-91.

[60] Wilhelm S, Carter C, Lynch M, et al. Discovery and development of sorafenib: a multikinase inhibitor for treating cancer. Nat Rev Drug Discov 2006;5(10):835-44.

[61] Thomas MB, Chadha R, Iwasaki $M$, et al. The combination of bevacizumab (B) and erlotinib (E) shows significant biological activity in patients with advanced hepatocellular carcinoma (HCC). 2007 ASCO Annual Meeting Proceedings. J Clin Oncol 2007; 25:18S (Abstr 4567). 